\title{
MULTIAXIAL INVESTIGATION OF PVC FOAMS AND ANALYSIS OF THE DEFORMATION MECHANISM BY 3D-DIC
}

\author{
Francesca Concas*, Stefan Diebels, Anne Jung
}

\author{
Saarland University, Institute of Applied Mechanics, Campus A4.2, 66123 Saarbrücken, Germany \\ * corresponding author: francesca.concas@uni-saarland.de
}

\begin{abstract}
Closed-cell polyvinylchloride (PVC) foams are widely used as core for sandwich composites for applications, in which multiaxial loads are involved. In the present work a wide range of uniaxial (tension, compression and torsion) and multiaxial experiments (both simultaneous tension-torsion and compression-torsion) were conducted on a high performance PVC foam. Failure data for each experiment were collected and depicted in the invariants plane. The whole cylindrical surface of the specimen was monitored by means of an 8-camera-system, strain fields were obtained by 3D-DIC. Hence, the occurrence and the evolution of deformation bands were inspected. The usage of an 8-camera system was essential for the observation of the deformation mechanism, especially for pure compression, pure torsion and combined axial load-torsion, in which the arising of deformation bands is affected by the occurrence of buckling and the orthotropy of the foam.
\end{abstract}

KeYwords: Polymeric foam, multiaxial testing, three-dimensional digital image correlation.

\section{INTRODUCTION}

Closed-cell polyvinylchloride (PVC) foams are broadly used as core material for sandwich composites in the marine industry [1] and for the construction of wind turbine blades [2]. In such applications, the core withstands multiaxial loads. Hence, a proper knowledge of the mechanical behaviour of the above-mentioned foams under multiaxial loading conditions is of great concern. This topic was addressed by several works, e.g. Christensen et al. [3] and Gdoutos et al. [4] conducted combined tension-torsion and compressiontorsion tests among other experiments on hollow cylindrical specimens made of PVC foams. In both previous works, adhesives were used to fix the specimen's position. The multiaxial behaviour of the same foam grade was investigated more recently by Shafiq et al. [5], who applied uniaxial, biaxial and triaxial loads by means of a specific testing device. Hoo Fatt et al. 6] carried out cyclic multiaxial tests on the same foam grade. In the present work uniaxial and multiaxial experiments were performed on a high performance PVC foam core grade. First, pure tension, pure compression and pure torsion were applied on the specimens. Failure loads were calculated for each experiment. Afterwards, simultaneous tensile-torsion experiments and simultaneous compression-torsion experiments were conducted by considering several percentages of the axial failure load. Failure stresses were then collected in the invariants plane for outlining the failure surface of the high performance PVC foam. The deformation of the specimen was inspected for some specific loading cases by means of the threedimensional digital image correlation (3D-DIC) and an 8-camera system. Such procedure allowed the monitoring of $360^{\circ}$ of the specimen.

\section{MATERIALS AND METHODS}

Experiments were done on the high performance Divinycell ${ }^{\circledast}$ HP100 PVC foams core grade (Diab AB, Laholm, Sweden) using an ElectroPuls ${ }^{\text {TM }}$ E10000 universal testing machine (Istron Ltd., Pfungstadt, Germany, load cell capacity $10 \mathrm{kN}$ for axial loading and $100 \mathrm{Nm}$ for torsional loading), which was equipped with two three-jaw chucks for clamping the cylindrical specimens. The 3D-DIC was achieved through the 8-camera system and the software Istra $4 \mathrm{D}$ v. 4.4 .2 (Dantec Dynamics GmbH, Skovlunde, Denmark). The eight cameras Manta G-235B (Allied Vision Technologies GmbH, Stadtroda, Germany) were disposed in aluminium bars in order to compose an octagon around the specimen. The experimental set-up is shown in Figure 1 (a). The maximal frame rate of the cameras was 50.8 fps. Images had a resolution of $1936 \times 1216$ pixels with a pixel size of $5.86 \mu \mathrm{m} \times 5.86 \mu \mathrm{m}$ and the acquisition rate was $1 \mathrm{fps}$. Three lamps were also needed for providing an illumination of the specimen with an adequate uniformity. The light sources consisted in two led bulbs and a halogen light source KL 2500 LCD (Carl Zeiss AG, Oberkochen, Germany) equipped with two flexible light guides. The specimen had a cylindrical gauge part with a diameter and a height of $25 \mathrm{~mm}$ and $50 \mathrm{~mm}$, respectively. Both ends of the specimen had a conical part and a shorter cylindrical part with a bigger diameter in order to allow the positioning of custom-made bushings as shown in Figure 1 (b). This method prevented the usage of adhesives for fixing the specimen's position. The same specimen was used for both uniaxial and multiaxial tests. Such geometry was manufactured by using the lathe with a grinding wheel as cutting tool. It is widely known that PVC foams are orthotropic because of the elongation of the cells towards the through-thickness 
(a)

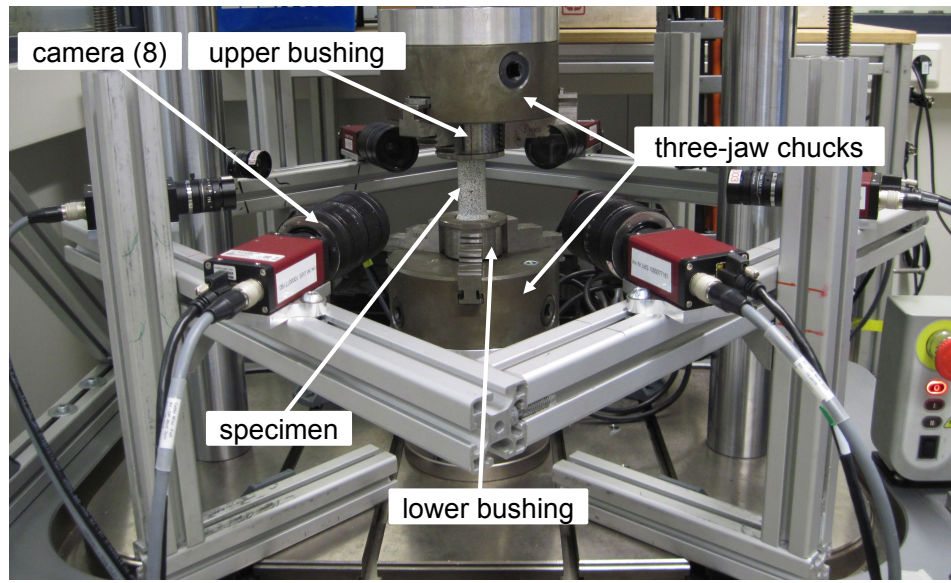

(b)

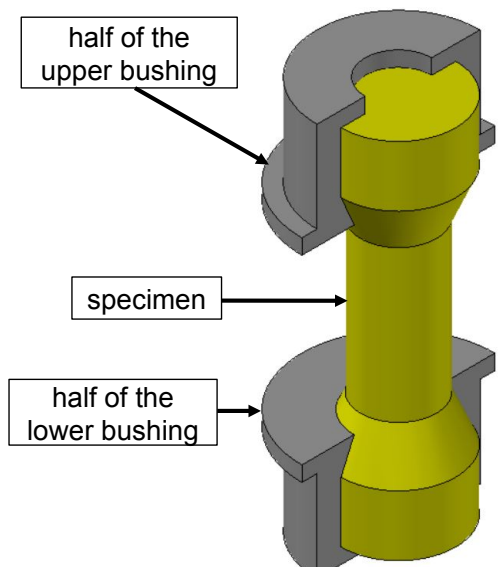

FiguRE 1. Set-up of the experiments (a) and cutaway of the bushing on the specimen (b).

direction 7, 8. Based on the thickness of the available panels, i.e. $40 \mathrm{~mm}$, the axis of the cylindrical specimen was perpendicular to the direction of cell elongation and hence the in-plane properties were evaluated by the experiments described in this work.

Tensile, compression and torsion experiments were conducted and failure loads are determined. Afterwards, combined tension-torsion and combined compression-torsion were performed by considering an increasing contribution of the axial load. The procedure for performing multiaxial tests is described as follows: the actuator of the universal testing machine applied the tensile or the compressive load to the specimen up to a percentage of the uniaxial failure load, i.e. $25 \%, 50 \%$ and $75 \%$ and a further percentage of $90 \%$ for combined tension-torsion tests. When the desired percentage was reached, the torsion was applied up to the rupture of the specimen or until reaching the limit switch, in case the specimen did not break. The reached tensile or compressive load was kept constant during the superposition of torsion, in order to make a simultaneous loading tension-torsion or compressiontorsion. Failure limits were calculated for combined tension-torsion and combined compression-torsion as well. For each loading case, three experiments were conducted for the sake of statistics. Finally, failure limits were collected in the invariants plane. Tensile and compression experiments were performed under displacement-control with a machine speed of 0.1 $\mathrm{mm} / \mathrm{s}$. The preload of multiaxial experiments was applied by load-control with a speed of $5 \mathrm{~N} / \mathrm{s}$. The application of torsion was done by rotational-control with an angular velocity of $0.5 \% \mathrm{~s}$ for pure torsion experiments as well as for multiaxial experiments. For the pure tensile, compression and torsion tests and for the combined tension-torsion and compression-torsion experiments with $50 \%$ of the axial failure load, strain fields were obtained by 3D-DIC and are displayed in the unwrapped cylindrical surface by means of the software h5pyViewer ${ }^{\odot}$ (Thierry Zamofing, Paul Scherrer Institute, Switzerland). DIC is a full-field optical method, which evaluates displacements and strains by tracking the motion of the specimen'speckle pattern during the experiment $[9$. The input values for the facet size and the grid spacing were 29 pixels and 3 pixels, respectively. The speckle pattern was applied as aerosol varnish on the specimens.

\section{Results And Discussion}

Stress-strain diagrams are shown in Figure 2 (a) for pure tensile tests and pure compression tests. Stresses and strains are reported as absolute values for comparing directly the compressive diagrams with the tensile diagrams. The occurrence of the buckling in the specimen during compression loading is highlighted in one of the diagrams in Fig. 2 (a). Figures 2 (b-d) show the shear stress-shear strain diagrams for pure torsion tests, for combined tensile-torsion tests and combined compression-torsion tests, respectively. For pure tension, pure torsion and combined tension-torsion, the specimen fractured at the end of the tests, then the load leading to the rupture of the specimen was considered as failure load. For pure compression and combined compression-torsion the specimen did not fracture, whereas a peak yield stress is evident in the diagrams. For such loading cases, the load leading the specimen to yielding was considered as failure load. Stresses and strains were calculated by the output file of the universal testing machine, hence the strains of Figure 2 are global strains, which are distinguished by local strains obtained by the 3D-DIC.

Afterwards, failure data were collected in the invariants plane, i.e. the first principal invariant and the squared root of the second deviatoric invariant (see Figure 3). The yield function proposed by Bier et al. 10 12 was adapted as failure criterion to the gathered failure data of the PVC foam. Further details on the fitting are given by Concas et al. 8

The arising and the evolution of deformation bands were inspected in the strain fields given by the 3D-DIC. For sake of conciseness, strain fields are shown only for two loading cases, i.e. pure 
(a)

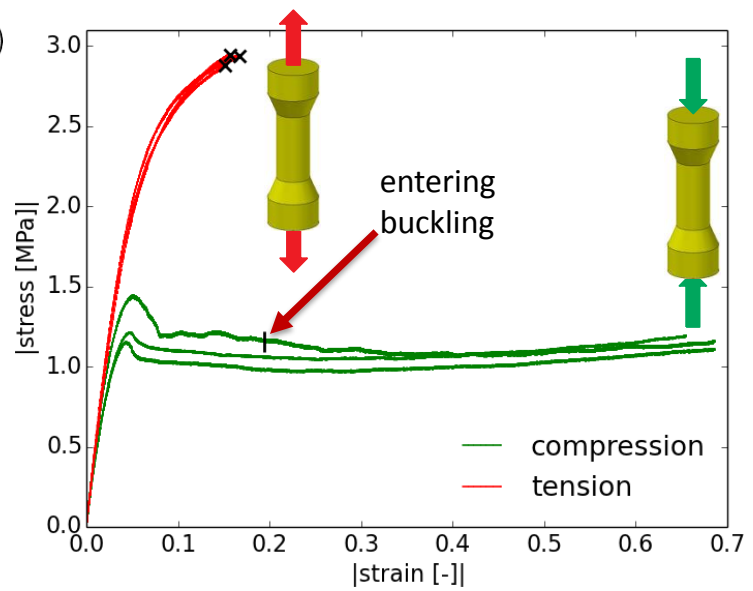

(c)



(b)

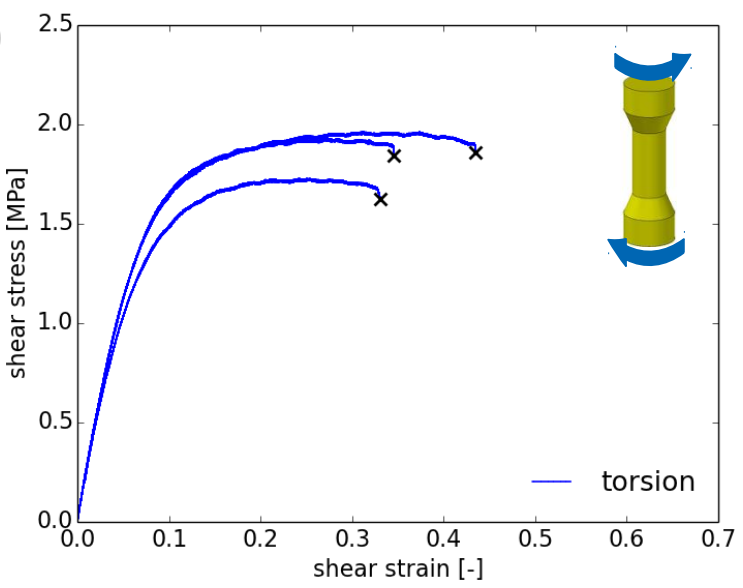

(d)

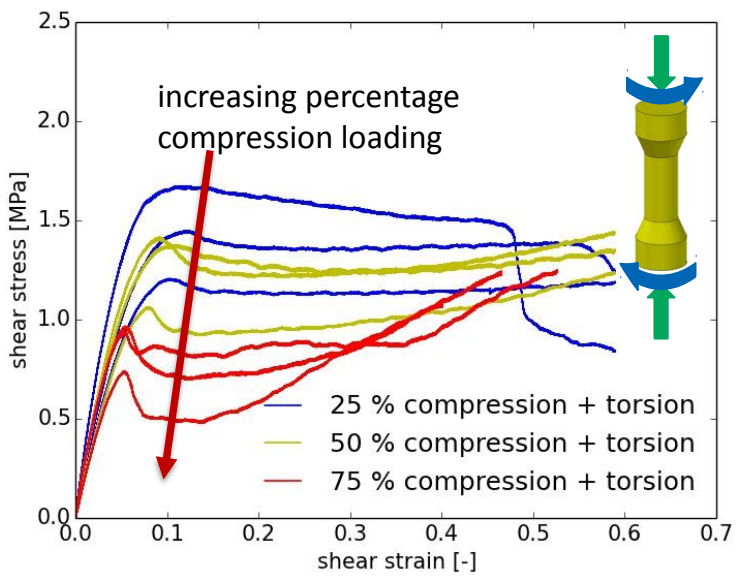

FiguRE 2. Stress-strain diagrams for the tension tests and the compression tests (a) and the shear stress-shear strain diagrams for the torsion tests (b), the combined tension-torsion tests (c) and the combined compression-torsion tests (d).

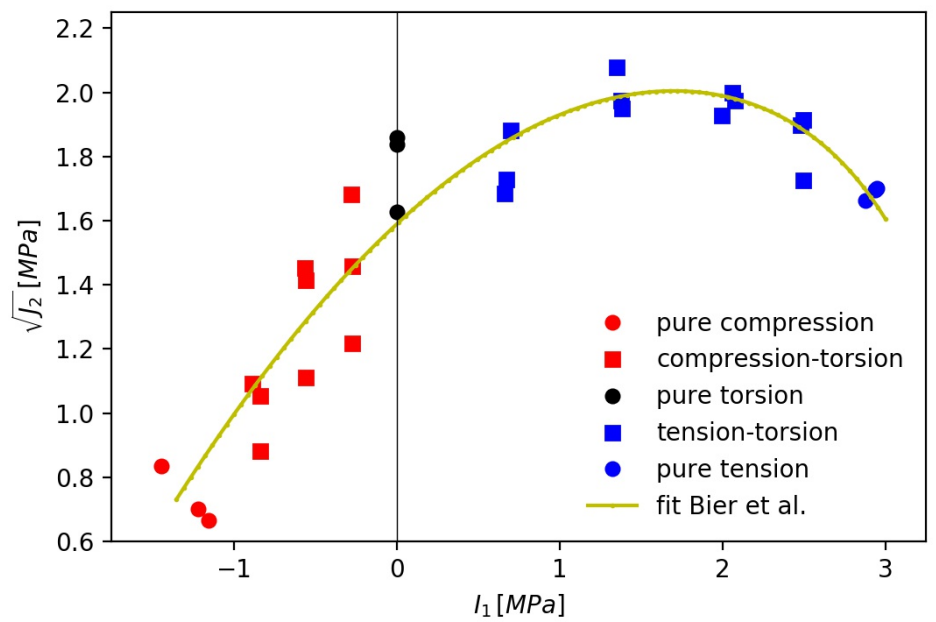

FIGURE 3. Failure data in the invariants plane and fitting of the failure criterion proposed by Bier et al. $10-12$. 



FiguRE 4. Local Lagrangian vertical strain fields with the corresponding absolute values of the global strain $|\varepsilon|$ for a pure compression test.

compression and combined tension-torsional loading, since they reflect the main typical characteristics of the strain maps of PVC foams for the other loading cases as well. Concerning the combined tension-torsion experiment, the axial load was kept constant to the $50 \%$ of the tensile failure load during the superposition of torsion. For each loading case, three strain fields are shown, which refer to three global strain values in the respective stress-strain diagram.

The local Lagrangian vertical strain fields are shown in Figure 4 for three absolute values of the global strain for a simple compression test. In this case, deformation bands were detected only when the plateau stage was reached. During the plateau, the specimen underwent a growing buckling. In the last strain field, areas with positive and negative local vertical strain values are distinguishable due to the severe buckling of the specimen, where local strains are positive in the convex part of the curved specimen and negative in the concave part.

Figure 5 shows the local Lagrangian shear strain fields for three global shear strain values of the combined tension-torsion test. In the second global strain stage of Figure 5 the positioning of deformation bands in two diametrically opposed areas of the specimen is observed. Such deformation bands are kept also in the last global strain stage's map of Figure 5 but it is less visible because of the arising of a deformation band with the highest strain gradient in the lower part of the specimen, where the fracture begins. This feature was observed also in pure tensile tests, whereas the gathering of deformation bands in two diametrically opposed wedges of the specimen was visible also in pure torsion tests and combined compression-torsion tests. In all tests, the widening black areas in the strain maps for the increasing global strains are due to the high distortion of the specimen and the consequent loss of the tracked pattern.

\section{Conclusions}

The present work described the execution of uniaxial and multiaxial tests on cylindrical specimens of the Divinycell ${ }^{\circledR} \mathrm{HP} 100$ PVC foam. The usage of the same geometry for all tests allowed a direct comparison between the different loading cases, i.e. the diagrams of Figures 2 (b-d). Hence, the increasing percentage of the axial failure load, either tension or compression, combined with a torsional load caused a lower failure limit in comparison to pure torsion tests. Failure data were calculated for all tests and collected in the invariants plane. The yield function of Bier et al. [10 12] was adapted successfully to the failure data of the PVC foam. Strain maps were obtained for the whole cylindrical surface of the specimen by using the 3D-DIC and the 8-camera-system. In this work, the strain fields were reported for two different 



$-0.08$

0.00

FigURE 5. Local Lagrangian shear strain fields with the corresponding global shear strain values $\gamma$ of a simultaneous tensile-torsion test, in which the $50 \%$ of the tensile failure load is reached.

loading cases, i.e. pure compression and combined tension-torsion. For the pure compression test, deformation bands were visible only when the specimen had entered the plateau stage. For higher global strains, deformation bands merged for composing areas with positive and negative local strain values, which were consistent with the curvature of the buckled specimen. For the combined tension-torsion test, deformation bands were mainly positioned in two diametrically opposed areas of the specimen due to the elongation of cells perpendicularly to the axis of the cylindrical specimen. The arising of a deformation band with the highest strain gradients allowed the prediction of the imminent fracture of the specimen. The usage of the 8-camera-system permitted a complete monitoring of deformation bands on the chosen specimen geometry. For the applied loading cases, the particular localisation of deformation bands could not be detected if a lower number of cameras were used. A 4-camerasystem is efficient for other specimen geometries, e.g. flat dog bone specimens [13, but it would redouble the extensions of black areas in the strain maps when the above-mentioned cylindrical geometry is observed.

\section{ACKNOWLEDGEMENTS}

The authors acknowledge Prof. F. Aymerich and Prof. A. Baldi (University of Cagliari, Italy) for useful discussions and for supplying the PVC foams panels. The University of Cagliari is also acknowledged for financial support.

\section{REFERENCES}

[1] J. Graham-Jones, J. Summerscales. Marine applications of advanced fibre-reinforced composites. Woodhead Publishing, 2016.

[2] O. Thomsen. Sandwich materials for wind turbine blades - present and future. Journal of Sandwich Structures \& Materials 11(1):7-26, 2009. DOI:10.1177/1099636208099710

[3] R. Christensen, D. Freeman, S. DeTeresa. Failure criteria for isotropic materials, applications to low-density types. International Journal of Solids and Structures 39(4):973-982, 2002. DOI:10.1016/S0020-7683(01)00229-3

[4] E. Gdoutos, I. Daniel, K.-A. Wang. Failure of cellular foams under multiaxial loading. Composites Part A: Applied Science and Manufacturing 33(2):163-176, 2002. DOI:10.1016/S1359-835X(01)00110-5

[5] M. Shafiq, R. Ayyagari, M. Ehaab, M. Vural. Multiaxial yield surface of transversely isotropic foams: Part II Experimental. Journal of the Mechanics and Physics of Solids 76:224-236, 2015. DOI:10.1016/j.jmps.2014.10.009

[6] M. Hoo Fatt, A. Jacob, X. Tong, A. MacHado-Reyes. Crushing behavior and energy absorption of PVC foam: an anisotropic visco-elastic-plastic-damage model. 21st International Conference on Composite Materials, Xían, 20-25th August 2017.

[7] S. Zhang, J. Dulieu-Barton, R. Fruehmann, O. T.

Thomsen. A methodology for obtaining material properties of polymeric foam at elevated temperatures. 
Experimental Mechanics 52(1):3-15, 2012. DOI:10.1007/s11340-011-9519-7.

[8] F. Concas, S. Diebels, A. Jung. Multiaxial failure surface of PVC foams and monitoring of deformation bands by three-dimensional digital image correlation. Journal of the Mechanics and Physics of Solids 130:195-215, 2019. DOI:10.1016/j.jmps.2019.06.008.

[9] A. Jung, K. Al Majthoub, C. Jochum, et al. Correlative digital image correlation and infrared thermography measurements for the investigation of the mesoscopic deformation behaviour of foams. Journal of the Mechanics and Physics of Solids 130:160-180, 2019. DOI:10.1016/j.jmps.2019.06.009

[10] W. Bier, S. Hartmann. A finite strain constitutive model for metal powder compaction using a unique and convex single surface yield function. European Journal of Mechanics-A/Solids 25(6):1009-1030, 2006. DOI:10.1016/j.euromechsol.2006.01.002.
[11] W. Bier, M. Daniel, N. Frage, et al. Die compaction of copper powder designed for material parameter identification. International Journal of Mechanical Sciences 49(6):766-777, 2007. DOI:10.1016/j.ijmecsci.2006.09.026

[12] S. Hartmann, W. Bier. High-order time integration applied to metal powder plasticity. International Journal of Plasticity 24(1):17-54, 2008. DOI:10.1016/j.ijplas.2007.01.014

[13] C. Röhrig, T. Scheffer, S. Diebels. Mechanical characterization of a short fiber-reinforced polymer at room temperature: experimental setups evaluated by an optical measurement system. Continuum Mechanics and Thermodynamics 29(5):1093-1111, 2017. 